\title{
Gray Codes Avoiding Matchings $\|^{\dagger}$
}

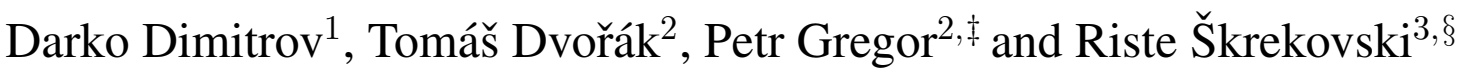 \\ ${ }^{1}$ Institut für Informatik, Freie Universität Berlin, Takustraße 9, 14195 Berlin, Germany. \\ ${ }^{2}$ Faculty of Mathematics and Physics, Charles University, Malostranské nám. 25, 11800 Praha, Czech Republic. \\ ${ }^{3}$ Department of Mathematics, University of Ljubljana, Jadranska 19, 1111 Ljubljana, Slovenia.
}

A (cyclic) $n$-bit Gray code is a (cyclic) ordering of all $2^{n}$ binary strings of length $n$ such that consecutive strings differ in a single bit. Equivalently, an $n$-bit Gray code can be viewed as a Hamiltonian path of the $n$-dimensional hypercube $Q_{n}$, and a cyclic Gray code as a Hamiltonian cycle of $Q_{n}$. In this paper we study (cyclic) Gray codes avoiding a given set of faulty edges that form a matching. Given a matching $M$ and two vertices $u, v$ of $Q_{n}, n \geq 4$, our main result provides a necessary and sufficient condition, expressed in terms of forbidden configurations for $M$, for the existence of a Gray code between $u$ and $v$ that avoids $M$. As a corollary, we obtain a similar characterization for a cyclic Gray code avoiding $M$. In particular, in the case that $M$ is a perfect matching, $Q_{n}$ has a (cyclic) Gray code that avoids $M$ if and only if $Q_{n}-M$ is a connected graph. This complements a recent result of Fink, who proved that every perfect matching of $Q_{n}$ can be extended to a Hamiltonian cycle. Furthermore, our results imply that the problem of Hamiltonicity of $Q_{n}$ with faulty edges, which is NP-complete in general, becomes polynomial for up to $2^{n-1}$ edges provided they form a matching.

Keywords: Gray code, hypercube, Hamiltonian path, Hamiltonian cycle, matching, fault tolerance

\section{Introduction}

A (cyclic) $n$-bit Gray code is a (cyclic) ordering of all $2^{n}$ binary strings of length $n$ such that consecutive strings differ in a single bit. It is named after Frank Gray, who in 1953 patented a simple scheme to generate such a cyclic code for every $n \geq 2$ [7]. Since then, the research on Gray codes satisfying certain additional properties has received a considerable attention, and applications have been found in such diverse areas as data compression, graphics and image processing, information retrieval, signal encoding or processor allocation in hypercubic networks [13].

Alternatively, an $n$-bit Gray code can be viewed as a Hamiltonian path of the $n$-dimensional hypercube $Q_{n}$, and a cyclic Gray code as a Hamiltonian cycle of $Q_{n}$. The applications of hypercubes in parallel computing [10] inspired the investigation of Hamiltonian paths and cycles in hypercubes avoiding a given set of faulty edges. Chan and Lee [2] proved that the problem whether $Q_{n}$ contains a Hamiltonian cycle avoiding a given set $\mathcal{F}$ of faulty edges is NP-complete. On the other hand, they showed that if $|\mathcal{F}| \leq$

\footnotetext{
${ }^{\dagger}$ Partially supported by the Czech-Slovenian bilateral grant MEB 090805.

¥Corresponding author, gregor@ktiml.mff.cuni.cz. Partially supported by the Czech Science Foundation Grant 201/08/P298.

$\S$ The work of this author was partially supported by Slovenian ARRS Research Grant P1-0297.
} 
$2 n-5, n \geq 3$, then such a Hamiltonian cycle exists if and only if each vertex is incident with at least two nonfaulty edges. This upper bound is sharp in the sense that for any $n \geq 3$ there are $2 n-4$ faulty edges in $Q_{n}$ satisfying the above condition, but there is no Hamiltonian cycle avoiding them. Tsai [14] obtained a similar result for Hamiltonian paths with given endvertices.

A related problem considers Hamiltonian cycles and paths in hypercubes passing through a set of prescribed edges. Caha and Koubek [1] observed that if a set $\mathcal{P}$ of prescribed edges extends to a Hamiltonian cycle, then it induces a subgraph consisting of pairwise vertex-disjoint paths. On the other hand, they showed that for any $n \geq 3$ there is a set of $2 n-2$ edges, satisfying this necessary condition, but there is no Hamiltonian cycle of $Q_{n}$ containing them. This bound is sharp in the sense that if $|\mathcal{P}| \leq 2 n-3$, $n \geq 2$, the necessary condition is also sufficient for a Hamiltonian cycle of $Q_{n}$ passing through every edge of $\mathcal{P}$ [3]. There is a similar result for Hamiltonian paths with prescribed endvertices if $|\mathcal{P}| \leq 2 n-4$, $n \geq 5$ [4]. A special case of the above problem, posed by Ruskey and Savage [12], is when the prescribed edges comprise a matching of the hypercube. Kreweras [9] conjectured that every perfect matching of $Q_{n}, n \geq 2$, can be completed to a Hamiltonian cycle. Recently, the conjecture was proved by Fink [5].

In this paper we study (cyclic) Gray codes avoiding a given set of faulty edges that form a matching. Given a matching $M$ and two vertices $u, v$ of $Q_{n}, n \geq 4$, our main result provides a necessary and sufficient condition for the existence of a Gray code between $u$ and $v$ that avoids $M$. As a corollary, we obtain a similar characterization for the existence of a cyclic Gray code avoiding $M$. In particular, in the case that $M$ is a perfect matching, $Q_{n}$ has a (cyclic) Gray code that avoids $M$ if and only if $Q_{n}-M$ is a connected graph.

Note that this complements the above quoted result of Fink. Furthermore, since our necessary and sufficient conditions are decidable in a polynomial time, our results imply that the problem of Hamiltonicity of $Q_{n}$ with faulty edges, which is NP-complete in general, becomes polynomial for up to $2^{n-1}$ edges provided they form a matching.

After definitions and preliminary results in the next two sections, we study necessary conditions in Sections 4 and 5. The induction step of our construction is presented in Section 6, while the obtained results are summarized in Section 7 The study of initial cases $n=3$ and $n=4$, which involves a rather elaborate case analysis, can be found in Appendix A and B.

\section{Preliminaries}

In this text $n$ is always a positive integer and $[n]$ denotes the set $\{1,2, \ldots, n\}$. As usual, the vertex and edge sets of a graph $G$ are denoted by $V(G)$ and $E(G)$, respectively. For a vertex $v \in V(G)$ and a set $E \subseteq E(G)$, let $G-v$ denote the subgraph of $G$ induced by $V(G) \backslash\{v\}$, and let $G-E$ denote the graph with vertices $V(G)$ and edges $E(G) \backslash E$. Similarly, for a pair of vertices $x, y \in V(G)$ let $G+x y$ denote the graph with vertices $V(G)$ and edges $E(G) \cup\{x y\}$. The distance of vertices $u$ and $v$ is denoted by $d(u, v)$. The distance $d(u, v w)$ of a vertex $u$ and an edge $v w$ is defined as the minimum of distances $d(u, v)$ and $d(u, w)$.

A path between $a$ and $b$, denoted by $P_{a b}$, is a graph consisting of pairwise distinct vertices $a=$ $x_{1}, x_{2}, \ldots, x_{n}=b$ and edges $x_{i} x_{i+1}$ for all $i \in[n-1]$. Note that if $a=b$, then $n=1$ and we obtain the trivial path $P_{a a}$ consisting of a single vertex $a$. If $P_{a b}$ and $P_{c d}$ are vertex disjoint paths and $b$ and $c$ are adjacent vertices, then $P_{a b}+P_{c d}$ denotes the path between $a$ and $d$, obtained as a concatenation of $P_{a b}$ with $P_{c d}$. A path $P$ is called a subpath of a path $P^{\prime}$ if $P$ forms a subgraph of $P^{\prime}$. We say that paths $\left\{P_{i}\right\}_{i=1}^{n}$ are spanning paths of a graph $G$ if $\left\{V\left(P_{i}\right)\right\}_{i=1}^{n}$ partitions $V(G)$. 
The $n$-dimensional hypercube $Q_{n}$ is the graph with vertex set $V\left(Q_{n}\right)=\{0,1\}^{n}$ and edge set $E\left(Q_{n}\right)=$ $\{u v|| \Delta(u, v) \mid=1\}$ where $\Delta(u, v)=\left\{i \in[n] \mid u_{i} \neq v_{i}\right\}$. The dimension $\operatorname{dim}(u v)$ of an edge $u v \in E\left(Q_{n}\right)$ is the integer $d$ such that $\Delta(u, v)=\{d\}$. For a vertex $v \in V\left(Q_{n}\right)$ let $v^{d}$ denote the vertex of $Q_{n}$ such that $v v^{d}$ is an edge of dimension $d$.

All edges of the same dimension $d$ form a layer (of dimension $d$ ) in $Q_{n}$. Note that $E\left(Q_{n}\right)$ is partitioned into $n$ layers, each of size $2^{n-1}$. Moreover, for every $d \in \Delta(u, v)$, each path $P_{u v}$ contains an edge of dimension $d$. In other words, vertices $u$ and $v$ are separated by the layer of dimension $d$.

The parity $p(v)$ of a vertex $v \in V\left(Q_{n}\right)$ is defined by $p(v)=\|v\| \bmod 2$ where $\|v\|=\sum_{i=1}^{n} v_{i}$. Note that vertices of each parity form bipartite sets of $Q_{n}$. Consequently, $p(u)=p(v)$ if and only if $d(u, v)$ is even. We extend the definition of the parity to edges by putting for $u v \in E\left(Q_{n}\right)$

$$
p(u v)= \begin{cases}p(u), & \text { if }\|u\|<\|v\| ; \\ p(v), & \text { otherwise. }\end{cases}
$$

For every $d \in[n]$ let $Q_{L}^{d}$ and $Q_{R}^{d}$ denote the subgraphs of $Q_{n}$ induced by the sets $\left\{v \in V\left(Q_{n}\right) \mid v_{d}=\right.$ $0\}$ and $\left\{v \in V\left(Q_{n}\right) \mid v_{d}=1\right\}$, respectively. The symbols $L$ and $R$ stand for 'left' and 'right', which corresponds to the presentation of $Q_{L}$ and $Q_{R}$ in our figures. Note that both $Q_{L}^{d}$ and $Q_{R}^{d}$ are isomorphic to $Q_{n-1}$. Similarly, for a matching $M$ in $Q_{n}$ put

$$
\begin{aligned}
& M^{d}=\{e \in M \mid \operatorname{dim}(e)=d\}, \\
& M_{L}^{d}=M \cap E\left(Q_{L}^{d}\right), \text { and } \\
& M_{R}^{d}=M \cap E\left(Q_{R}^{d}\right) .
\end{aligned}
$$

Observe that $\left(Q_{i}^{a}\right)_{j}^{b}=\left(Q_{j}^{b}\right)_{i}^{a}$ and $\left(M_{i}^{a}\right)_{j}^{b}=\left(M_{j}^{b}\right)_{i}^{a}$ for every $a, b \in[n]$ and $i, j \in\{L, R\}$.

Given a set $F$ of faulty edges of $Q_{n}$, we say that a subgraph of $Q_{n}$ avoids $F$ if it forms a subgraph of $Q_{n}-F$.

\section{Some fundamental results}

A classical result of Lewinter and Widulski [11] describes Hamiltonian paths in hypercubes with one faulty vertex.

Lemma 1 For any pairwise distinct vertices $u, v, w$ in $Q_{n}$ such that $p(u)=p(v) \neq p(w)$, there exists a Hamiltonian path of $Q_{n}-w$ between $u$ and $v$.

There is a similar characterization for hypercubes with at most one faulty edge, which follows from more general results of Tsai et al. [15]. It should be remarked that the special case of $M=\emptyset$ was firstly observed by Havel [8].

Lemma 2 For every $n \geq 3$, vertices $u$ and $v$ of different parities, and a matching $M$ of $Q_{n}$ with $|M| \leq 1$, there exists a Hamiltonian path in $Q_{n}-M$ between $u$ and $v$.

An extension of the above mentioned Havel's result was obtained in [3].

Lemma 3 For every distinct vertices $u, v, x, y$ of $Q_{n}$ such that $p(u) \neq p(v)$ and $p(x) \neq p(y)$, there exist spanning paths $P_{u v}, P_{x y}$ of $Q_{n}$.

We shall also employ the following lemma on the existence of a Hamiltonian path passing through a prescribed edge, which is a special case of more general results of Caha and Koubek [1]. 
Lemma 4 For every two vertices $u, v$ and an edge e of $Q_{n}$ such that $p(u) \neq p(v)$ and $e \neq u v$, there exists a Hamiltonian path of $Q_{n}$ between $u$ and $v$ passing through $e$.

We conclude this section with a simple observation.

Proposition 1 For every matching $M$ in $Q_{n}$ with $|M|=2$ there exists a dimension $d \in[n]$ such that $\left|M_{L}^{d}\right|=\left|M_{R}^{d}\right|=1$.

Proof: We argue by induction on $n$. Since the case $n \leq 2$ is obvious, assume that $n \geq 3$ and choose an arbitrary $d \in[n] \backslash\{\operatorname{dim}(e) \mid e \in M\}$. If $\left|M_{L}^{d}\right|=\left|M_{R}^{d}\right|=1$, we are done. Otherwise it must be the case that $M=M_{i}^{d}$ for some $i \in\{L, R\}$ and hence by the induction hypothesis, there exists $d^{\prime} \in[n] \backslash\{d\}$ such that $\left|M_{L}^{d^{\prime}}\right|=\left|\left(M_{i}^{d}\right)_{L}^{d^{\prime}}\right|=1=\left|\left(M_{i}^{d}\right)_{R}^{d^{\prime}}\right|=\left|M_{R}^{d^{\prime}}\right|$ as required.

\section{Half-layers}

A matching $M$ in $Q_{n}, n \geq 2$, is called a half-layer in $Q_{n}$ if $M$ consists exactly of all edges of dimension $d$ and parity $p$ for some $d \in[n]$ and $p \in\{0,1\}$. Observe that each layer is partitioned into two half-layers, each of size $2^{n-2}$. Moreover, if a perfect matching of $Q_{n}$ contains a half-layer, then it actually is a layer of $Q_{n}$.

We shall see in the next section that half-layers are obstacles that cannot be overcome. More precisely, we shall see in Lemma 6 that there is no Hamiltonian cycle avoiding a half-layer. The need to treat half-layers as a special case motivates the following definitions.

Given a matching $M$ in $Q_{n}$, we call $d \in[n]$

- a main dimension of $M$ in $Q_{n}$ if $M^{d}$ contains a half-layer in $Q_{n}$;

- a splitting dimension for $M$ if $M_{i}^{d}$ does not contain a half-layer in $Q_{i}^{d}$ for both $i \in\{L, R\}$.

Now we show that the main dimension is uniquely determined.

Proposition 2 Let $d$ be a main dimension of a matching $M$ in $Q_{n}, n \geq 2$. Then,

(i) $M$ contains only edges of dimension d. Consequently, $d$ is the only main dimension of $M$.

(ii) $d$ is the unique main dimension of $M_{i}^{a}$ in $Q_{i}^{a}$ for every $a \in[n] \backslash\{d\}$ and $i \in\{L, R\}$ provided $n \geq 3$.

Proof: Since for every edge $u v \in E\left(Q_{n}\right)$ of dimension $d^{\prime} \neq d$ we have $p(u) \neq p(v)$, it follows that at least one of $u, v$ must be incident with an edge of $M^{d}$. Hence $u v \notin M$ and consequently, $M_{L}^{d}=M_{R}^{d}=\emptyset$. This, together with the fact that $M^{d}$ contains a half-layer and therefore must be nonempty, verifies part $(i)$.

To see why $(i i)$ holds, note that if $n \geq 3$, then a half-layer in $Q_{n}$ contains a half-layer in both $Q_{L}^{a}$ and $Q_{R}^{a}$ by definition. It follows that $d$ is a main dimension of $M_{i}^{a}$ in $Q_{i}^{a}$ while the uniqueness follows from part $(i)$.

A matching $M$ in $Q_{n}$ is called aligned if there exists a dimension $d \in[n]$ such that $\left|M_{L}^{d}\right| \leq 1$ and $\left|M_{R}^{d}\right| \leq 1$. Otherwise, it is called unaligned. Note that a matching containing a half-layer is always aligned by Proposition $2(i)$. For aligned matchings our problem is easy, as we shall see in the next section. But this is not the case with unaligned matchings. For them, we use the following lemma. 
Lemma 5 If $M$ is an unaligned matching in $Q_{n}, n \geq 4$, then there are at least two splitting dimensions for $M$.

Proof: First note that if every $a \in[n]$ is a splitting dimension for $M$, we are done. Hence we can assume that this is not the case, i. e., there exist distinct $a, b \in[n]$ such that $b$ is the main dimension of $M_{L}^{a}$ in $Q_{L}^{a}$.

Case 1: $b$ is not a splitting dimension for $M$. Then there exist $i \in\{L, R\}$ and $d \in[n] \backslash\{b\}$ such that $d$ is the main dimension of $M_{i}^{b}$. Assume that $d \neq a$. Then Proposition 2 (ii) implies that $d$ is also the main dimension of $\left(M_{i}^{b}\right)_{L}^{a}$ in $\left(Q_{i}^{b}\right)_{L}^{a}$. It follows that $\left(M_{L}^{a}\right)_{i}^{b}=\left(M_{i}^{b}\right)_{L}^{a}$ contains a half-layer. On the other hand, as $b$ is the main dimension of $M_{L}^{a}$, Proposition $2(i)$ reveals that $M_{L}^{a}$ contains only edges of dimension $b$ and therefore $\left(M_{L}^{a}\right)_{i}^{b}=\emptyset$. This, however, leads to a contradiction since a half-layer is always nonempty. Hence we conclude that $d=a$.

Now choose an arbitrary $c \in[n] \backslash\{a, b\}$ and $j \in\{L, R\}$ and suppose that $M_{j}^{c}$ contains a half-layer in $Q_{j}^{c}$. Using Proposition 2 (ii) again, $a$ and $b$ are the main dimensions of $\left(M_{i}^{b}\right)_{j}^{c}$ and $\left(M_{L}^{a}\right)_{j}^{c}$ in $\left(Q_{i}^{b}\right)_{j}^{c}$ and $\left(Q_{L}^{a}\right)_{j}^{c}$, respectively. Since $\left(M_{i}^{b}\right)_{j}^{c}=\left(M_{j}^{c}\right)_{i}^{b}$ and $\left(M_{L}^{a}\right)_{j}^{c}=\left(M_{j}^{c}\right)_{L}^{a}$, part $(i i)$ of Proposition 2 implies that $a$ and $b$ are also main dimensions of $M_{j}^{c}$ in $Q_{j}^{c}$, while part $(i)$ then reveals that $a=b$, which is a contradiction with our assumption. Hence $c$ must be a splitting dimension for $M$. It follows that there exist $n-2 \geq 2$ splitting dimensions in this case.

Case 2: $b$ is a splitting dimension for $M$. In this case we need to show that there exists another splitting dimension for $M$, different from $b$. Assume, by way of contradiction, that this is not the case. Then for any $c \in[n] \backslash\{b\}$ there exists $i \in\{L, R\}$ such that $M_{i}^{c}$ contains a half-layer. Recall that $b$ is the main dimension of $M_{L}^{a}$ in $Q_{L}^{a}$. If $c \neq a$, then Proposition $2(i i)$ implies that $b$ is the main dimension of $\left(M_{L}^{a}\right)_{i}^{c}=\left(M_{i}^{c}\right)_{L}^{a}$ in $\left(Q_{L}^{a}\right)_{i}^{c}$ and therefore $b$ is also the main dimension of $M_{i}^{c}$ in $Q_{i}^{c}$. Hence we conclude that for any $c \in[n] \backslash\{b\}$ there exists $i \in\{L, R\}$ such that $b$ is the main dimension of $M_{i}^{c}$ in $Q_{i}^{c}$.

Since $M$ is an unaligned matching, there must exist $i \in\{L, R\}$ such that $M_{i}^{b}$ contains two distinct edges $e_{1}, e_{2}$. Note that both $\operatorname{dim}\left(e_{1}\right)$ and $\operatorname{dim}\left(e_{2}\right)$ are different from $b$. By Proposition 1 there must be a dimension $d \in[n] \backslash\{b\}$ such that $\left|M_{j}^{d} \cap\left\{e_{1}, e_{2}\right\}\right|=1$ for each $j \in\{L, R\}$. But then each $M_{j}^{d}$ contains an edge of dimension distinct from $b$, which by Proposition $2(i)$ means that $b$ cannot be the main dimension of $M_{j}^{d}$, contrary to the conclusion of the previous paragraph.

\section{Admissible configurations}

A nonempty matching $M$ is called an almost-layer (of dimension $d$ ) in $Q_{n}$ if there exist vertices $u, v \in Q_{L}^{d}$ of different parities such that $M$ consists of all edges of dimension $d$ except $u u^{d}$ and $v v^{d}$. Pairs $\{u, v\}$ and $\left\{u^{d}, v^{d}\right\}$ are called the free pairs of $M$.

The next lemma gives necessary conditions for a Gray code avoiding a given matching.

Lemma 6 Let $M$ be a matching and $u, v$ vertices of $Q_{n}$. If there exists a Hamiltonian path in $Q_{n}-M$ between $u$ and $v$, then

(i) $p(u) \neq p(v)$;

(ii) for every $d \in \Delta(u, v)$ there exists an edge e $\in E\left(Q_{n}\right) \backslash M$ of dimension $d$ such that $d(u, e)$ is odd;

(iii) for every $d \in[n] \backslash \Delta(u, v)$ there exist two edges in $E\left(Q_{n}\right) \backslash M$ of dimension d of different parities;

(iv) $M$ contains no almost-layer with a free pair $\{u, v\}$. 
Proof: Claims $(i)-($ iii $)$ follow from the facts that in a hypercube the number of vertices of odd parity equals the number of vertices of even parity, and that the parity of vertices on every path alternates. To verify $(i v)$, observe that if $M$ is an almost-layer with a free pair $\{u, v\} \subseteq V\left(Q_{L}^{d}\right)$, then every path $P_{u v}$ in $Q_{n}-M$ avoids either all vertices of $V\left(Q_{L}^{d}\right) \backslash\{u, v\}$, or all vertices of $V\left(Q_{R}^{d}\right)$, and therefore cannot be Hamiltonian.

We say that a triple $(u, v, M)$ is a configuration in $Q_{n}$ if $M$ is a matching in $Q_{n}$ and $u$ and $v$ are vertices of different parities, i. e., condition $(i)$ of Lemma 6 holds. Since we do not need to distinguish between vertices $u$ and $v$, we consider the configurations $(u, v, M)$ and $(v, u, M)$ to coincide. If, moreover, conditions $(i i)-(i v)$ of Lemma 6 hold, we say that the configuration $(u, v, M)$ is admissible.

The conditions ( ii) and (iii) of Lemma 6 can be unified into one condition using a slight modification of the concept of a half-layer. Note that since a half-layer contains only edges of the same parity, the distances of a given vertex to edges of a given half-layer must also have the same parity. If the distance of a vertex $u$ to edges of a half-layer $M$ in $Q_{n}$ is odd, we say that $M$ is an odd half-layer for the vertex $u$.

Proposition 3 A configuration $(u, v, M)$ is admissible if and only if the following holds

(i) $M$ contains no odd half-layer for $u$ or $v$; and

(ii) $M$ contains no almost-layer with a free pair $\{u, v\}$.

The next lemma shows that these conditions are also sufficient for the existence of a Hamiltonian path in $Q_{n}, n \geq 4$, provided that $M$ is aligned.

Lemma 7 Let $M$ be an aligned matching, and let $u$ and $v$ be vertices of $Q_{n}, n \geq 4$. If $(u, v, M)$ is an admissible configuration, then $Q_{n}-M$ contains a Hamiltonian path between $u$ and $v$.

Proof: Since $M$ is an aligned matching, there exists $d \in[n]$ such that $\left|M_{L}^{d}\right| \leq 1$ and $\left|M_{R}^{d}\right| \leq 1$. Without a loss of generality assume that $u \in V\left(Q_{L}^{d}\right)$ and consider the following two cases:

Case 1: $v \in V\left(Q_{R}^{d}\right)$. Since $(u, v, M)$ is an admissible configuration, there exists a vertex $w \in V\left(Q_{L}^{d}\right)$ such that $p(u) \neq p(w)$ and $w w^{d} \notin M$. Note that then also $p\left(w^{d}\right) \neq p(v)$. Now, by Lemma 2 , there exist Hamiltonian paths $P_{u w}$ of $Q_{L}^{d}-M_{L}^{d}$ and $P_{w^{d} v}$ of $Q_{R}^{d}-M_{R}^{d}$. Then $P_{u v}:=P_{u w}+P_{w^{d} v}$ is the required Hamiltonian path.

Case 2: $v \in V\left(Q_{L}^{d}\right)$. Here, we consider the following subcases.

Subcase 2.1: $M_{L}^{d}=\emptyset$. Since $(u, v, M)$ is an admissible configuration, there are vertices $x, y \in V\left(Q_{L}^{d}\right)$ such that $p(x) \neq p(y), x x^{d} \notin M, y y^{d} \notin M$, and $\{u, v\} \neq\{x, y\}$. Apply Lemma 2 to obtain a Hamiltonian path $P_{x^{d} y^{d}}$ of $Q_{R}^{d}-M_{R}^{d}$. Note that as $p(u) \neq p(v)$ and $p(x) \neq p(y)$, we can assume that also $p(u) \neq p(x)$ and $p(v) \neq p(y)$, interchanging $x$ and $y$ if necessary.

We conclude the subcase with the following construction. If $\{u, v\} \cap\{x, y\}=\emptyset$, then apply Lemma 3 to obtain spanning paths $P_{u x}$ and $P_{v y}$ of $Q_{L}^{d}$ and put $P_{u v}:=P_{u x}+P_{x^{d} y^{d}}+P_{y v}$. Otherwise it must be the case that $|\{u, v\} \cap\{x, y\}|=1$. Assume without a loss of generality that $v=x$ and $u \neq y$. Then apply Lemma 1 to obtain a Hamiltonian path $P_{u y}$ of $Q_{L}^{d}-v$ and put $P_{u v}:=P_{u y}+P_{y^{d} x^{d}}+P_{x x}$. In both cases we obtained the required Hamiltonian path.

Subcase 2.2: $M_{L}^{d}=\{e\}$. If $e \neq u v$, then put $x y=e$ and apply the construction of Subcase 2.1. So, we may assume that $e=u v$. Since $(u, v, M)$ is an admissible configuration, there is a vertex $y \in$ $V\left(Q_{L}^{d}\right) \backslash\{u, v\}$ such that $y y^{d} \notin M$. Assume without a loss of generality that $p(u) \neq p(y)$. Finally, put $x=v$ and apply the construction of Subcase 2.1 in order to conclude this case. 
Note that the above lemma does not hold for $n=3$, see e. g. configurations (e), (f) or (g) in Figure 1 . The case of small dimensions is analyzed separately in Appendix A and B.

\section{Gray codes in hypercubes of higher dimensions}

In this section we use $\operatorname{AL}\left(u, v, M, Q_{n}\right)$ to denote the fact that a matching $M$ contains an almost-layer in $Q_{n}$ with a free pair $\{u, v\}$. Recall that if $\operatorname{AL}\left(u, v, M, Q_{n}\right)$ holds, then there exists $d$ such that $M^{d}$ consists of all edges of dimension $d$ except $u u^{d}$ and $v v^{d}$. The following simple observation is used later.

Proposition 4 Let $M$ be a matching which contains an almost-layer $A$ with a free pair $\{u, v\}$ in $Q_{n}$, $n \geq 4$.

(i) If $\mathrm{AL}\left(u, w, M, Q_{n}\right)$ holds, then $v=w$.

(ii) If at least one of vertices $x, y$ is incident with an edge of $A$, then $\operatorname{AL}\left(x, y, M, Q_{n}\right)$ does not hold.

Note that the assumption $n \geq 4$ is for part $(i i)$ essential, as it fails to hold in the case that $M$ is the perfect matching of $Q_{3}$ depicted in Figure 2(10). Now we are ready to resolve the case of matchings not containing a half-layer.

Lemma 8 Let $u, v \in V\left(Q_{n}\right), n \geq 4$, such that $p(u) \neq p(v)$ and let $M$ be a matching in $Q_{n}$ containing no half-layer. Then $Q_{n}-M$ contains a Hamiltonian path between $u$ and $v$ unless $M$ is an almost-layer in $Q_{n}$ with a free pair $\{u, v\}$.

Proof: We argue by induction on $n$. Since the case $n=4$ is settled by Lemma 14 in Appendix B, assume that $n>4$. If $M$ is aligned, the statement follows from Lemma 7 If $M$ is unaligned, then by Lemma 5, there is $d \in[n]$ such that $M_{i}^{d}$ contains no half-layer in $Q_{i}^{d}$ for both $i \in\{L, R\}$. Without a loss of generality assume that $u \in V\left(Q_{L}^{d}\right)$. To obtain the desired Hamiltonian path $P_{u v}$ of $Q_{n}-M$, consider the following two cases:

Case 1: $v \in Q_{R}^{d}$. Since $M$ does not contain a half-layer, there is a vertex $w \in V\left(Q_{L}^{d}\right)$ such that

$$
p(u) \neq p(w) \quad \text { and } \quad w w^{d} \notin M
$$

Notice that also $p\left(w^{d}\right) \neq p(v)$. We claim that $w$ can be chosen so that neither $\operatorname{AL}\left(u, w, M_{L}^{d}, Q_{L}^{d}\right)$ nor $\operatorname{AL}\left(w^{d}, v, M_{R}^{d}, Q_{R}^{d}\right)$ holds. Indeed, if $M_{L}^{d}$ contains an almost-layer $A$ in $Q_{L}^{d}$ with a free pair $\{u, w\}$, then each edge of $A$ contains one vertex that can play the role of $w$ in 11 . Hence there are still $|A|=$ $2^{n-2}-2>2$ choices for $w$, and $M_{R}^{d}$ may contain an almost-layer in $Q_{R}^{d}$ with a free pair $\left\{w^{d}, v\right\}$ for no more than one of them by Proposition $4(i)$.

It remains to apply the induction to obtain Hamiltonian paths $P_{u w}$ and $P_{w^{d} v}$ of $Q_{L}^{d}-M_{L}^{d}$ and $Q_{R}^{d}-M_{R}^{d}$, respectively, and finally put $P_{u v}:=P_{u w}+P_{w^{d} v}$ to conclude this case.

Case 2: $v \in V\left(Q_{L}^{d}\right)$. Consider the following subcases.

Subcase 2.1: $\mathrm{AL}\left(u, v, M_{L}^{d}, Q_{L}^{d}\right)$ holds. Since $M_{L}^{d}$ contains an almost-layer $A$ with $2^{n-2}-2>3$ edges, Proposition $4(i i)$ implies that there must be an edge $x y \in A$ such that $M_{R}^{d}$ contains no almost-layer in $Q_{R}^{d}$ with a free pair $\left\{x^{d}, y^{d}\right\}$. Thus, by the induction hypothesis, there are Hamiltonian paths $P_{u v}$ and $P_{x^{d} y^{d}}$ of $Q_{L}^{d}-\left(M_{L}^{d} \backslash\{x y\}\right)$ and $Q_{R}^{d}-M_{R}^{d}$, respectively. Note that by Lemma 6, the path $P_{u v}$ passes through the edge $x y$, and hence there are subpaths $P_{u x}$ and $P_{y v}$ of $P_{u v}$ such that $P_{u v}=P_{u x}+P_{y v}$. It remains to put $P_{u v}:=P_{u x}+P_{x^{d} y^{d}}+P_{y v}$ to complete this subcase. 
Subcase 2.2: $\mathrm{AL}\left(u, v, M_{L}^{d}, Q_{L}^{d}\right)$ does not hold. First apply the induction hypothesis to obtain a Hamiltonian path $P_{u v}^{L}$ of $Q_{L}^{d}-M_{L}^{d}$. Next, distinguish the following two possibilities.

Subcase 2.2.1: $\left\{x x^{d}\right.$, yy $\left.y^{d}\right\} \cap M=\emptyset$ for some pair $x$, y of neighbors on $P_{u v}^{L}$. We claim that vertices $x$ and $y$ can be chosen so that $\mathrm{AL}\left(x^{d}, y^{d}, M_{R}^{d}, Q_{R}^{d}\right)$ does not hold. To verify the claim, suppose, by way of contradiction, that $M_{R}^{d}$ contains an almost-layer $A$ in $Q_{R}^{d}$ with a free pair $\left\{x^{d}, y^{d}\right\}$. Denote by $S$ the set of vertices $z$ of $Q_{L}^{d}$ such that $z^{d}$ is incident with an edge of $A$. Then

$$
|S|=2|A|=2^{n-1}-4>2^{n-2}=\left|V\left(Q_{L}^{d}\right)\right| / 2=\left|V\left(P_{u v}^{L}\right)\right| / 2
$$

and hence there exist $x, y \in S$ that are neighbors on $P_{u v}^{L}$. Then, it follows by Proposition $4(i i)$ that $\mathrm{AL}\left(x^{d}, y^{d}, M_{R}^{d}, Q_{R}^{d}\right)$ does not hold.

As in Subcase 2.1, it remains to apply the induction to obtain a Hamiltonian path $P_{x^{d} y^{d}}$ of $Q_{R}^{d}-M_{R}^{d}$ and put $P_{u v}:=P_{u x}+P_{x^{d} y^{d}}+P_{y v}$, where $P_{u x}$ and $P_{y v}$ are the subpaths of $P_{u v}^{L}$ satisfying $P_{u v}^{L}=P_{u x}+P_{y v}$.

Subcase 2.2.2: $\left\{x x^{d}, y y^{d}\right\} \cap M \neq \emptyset$ for each pair $x, y$ of neighbors on $P_{u v}^{L}$. By Lemma 5 there exists another splitting dimension $d^{\prime} \neq d$ and we claim that Subcase 2.2.2 for $d^{\prime}$ cannot occur. To verify the claim, first observe that

$$
\left|M^{d}\right| \geq\left|V\left(P_{u v}^{L}\right)\right| / 2=2^{n-2} .
$$

If this inequality is strict, then $|M| \leq 2^{n-1}$ implies that $\left|M^{d^{\prime}}\right|<2^{n-2}$, contrary to 2, and hence the claim holds. Thus we can assume that

$$
\left|M^{d}\right|=\left|M^{d^{\prime}}\right|=2^{n-2} .
$$

This means that $\left|M^{d}\right|=\left|V\left(P_{u v}^{L}\right)\right| / 2$, which together with the assumption of this subcase implies that $\left|\left\{x x^{d}, y y^{d}\right\} \cap M^{d}\right|=1$ for each pair $x, y$ of neighbors on $P_{u v}^{L}$. Since the parity of consecutive vertices on $P_{u v}^{L}$ alternates, it follows that if $u u^{d} \in M^{d}$, then $x x^{d} \in M^{d}$ for each vertex $x \in V\left(P_{u v}^{L}\right)$ with $p(u)=p(x)$. However, this would mean that $M$ contains a half-layer, contrary to our assumption. Since the same reasons apply to the endvertex $v$, we conclude that neither $u$ nor $v$ are incident with an edge of $M^{d}$. Applying the same argument to the dimension $d^{\prime}$, it follows that

$$
\left|M^{d}\right|+\left|M^{d^{\prime}}\right| \leq\left|V\left(Q_{n}\right) \backslash\{u, v\}\right| / 2=2^{n-1}-1,
$$

contrary to 3 .

\section{The main results}

Now we are ready to formulate the necessary and sufficient conditions for (cyclic) Gray codes avoiding a given matching. Note that the conditions are expressed in terms of half-layers and almost-layers, which were defined in Sections 4 and 5 , respectively.

Theorem 1 Let $M$ be a matching, and let $u$ and $v$ be vertices of $Q_{n}, n \geq 4$. Then $Q_{n}$ has a Gray code between $u$ and $v$ that avoids $M$ if and only if $M$ contains no odd half-layer for $u$ or $v$ and no almost layer with a free pair $\{u, v\}$.

Proof: Recall that $(u, v, M)$ is an admissible configuration if and only if the left side of the equivalence holds, by Proposition 3 The necessity is settled by Lemma 6 . The sufficiency follows from Lemma 7 in the case $M$ contains a half-layer, and from Lemma 8 otherwise. 
It should be noted that for $n=3$ the problem is fully described by Lemma 9 in Appendix A, and for $n \leq 2$ it is trivial.

Corollary 1 Let $M$ be a matching in $Q_{n}, n \geq 4$. Then $Q_{n}$ has a cyclic Gray code that avoids $M$ if and only if $M$ does not contain a half-layer.

Proof: Observe that the following two equivalences hold.

- $Q_{n}-M$ contains a Hamiltonian cycle $\Longleftrightarrow Q_{n}-M$ contains a Hamiltonian path $P_{u v}$ for some edge $u v \in E\left(Q_{n}\right)$;

- there exists an admissible configuration $(u, v, M)$ for some edge $u v \in E\left(Q_{n}\right) \Longleftrightarrow M$ does not contain a half-layer.

The corollary now follows from Theorem 1

Corollary 2 Let $M$ be a perfect matching in $Q_{n}$. Then the following three statements are equivalent:

(i) $Q_{n}$ has a Gray code avoiding $M$;

(ii) $Q_{n}$ has a cyclic Gray code avoiding $M$;

(iii) $Q_{n}-M$ is connected.

Proof: First observe that clearly $(i i) \Longrightarrow(i)$ and $(i) \Longrightarrow($ iii $)$. Hence it only remains to prove that $($ iii $) \Longrightarrow($ ii $)$. To that end, notice that for any perfect matching $M$ of $Q_{n}$,

$M$ contains a half-layer in $Q_{n} \Longleftrightarrow M$ forms a layer of $Q_{n} \Longleftrightarrow Q_{n}-M$ is disconnected.

The validity of the implication $($ iii $) \Longrightarrow($ ii $)$ for $n \geq 4$ now follows from Corollary 1 , the case $n=3$ may be verified by inspection of parts (9) and (10) of Figure 2 , and the case $n \leq 2$ is trivial.

\section{Appendix A: Gray codes in $Q_{3}$}

At this point it only remains to settle the initial cases $n=3$ and $n=4$. Note that we were able to use computer search to verify the main result of this part, namely Lemma 14, which is needed for the induction basis in the proof of Lemma 8 However, considering the difficulties in independently verifying computer-aided proofs, here we provide arguments that may be verified without a computer assistance. This involves a rather elaborate case analysis, but we believe that in this case it is indispensable.

A configuration $(u, v, M)$ in $Q_{n}$ is good if there is a Hamiltonian path of $Q_{n}-M$ between $u$ and $v$. Otherwise it is bad. In this terminology, our aim is to characterize all good configurations.

A configuration $\left(u, v, M_{1}\right)$ contains a configuration $\left(u, v, M_{2}\right)$ if $M_{1} \supseteq M_{2}$. A bad configuration is minimal if it does not contain any other bad configuration. An admissible configuration is maximal if it is not contained in any other admissible configuration. Similarly, a matching is maximal if it is not contained in any other matching.

We start with the list of all minimal bad configurations in $Q_{3}$.

Lemma 9 Let $u$ and $v$ be vertices of different parity and let $M$ be a matching in $Q_{3}$. Then there is a Hamiltonian path of $Q_{3}-M$ between $u$ and $v$ unless $(u, v, M)$ contains one of the bad configurations depicted in Figure 1 


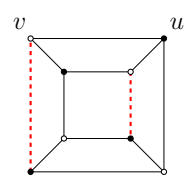

(a)

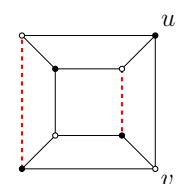

(b)

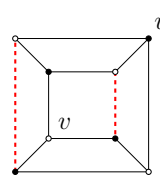

(c)

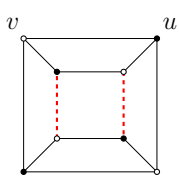

(d)

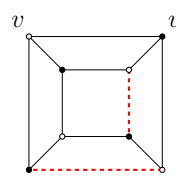

(e)

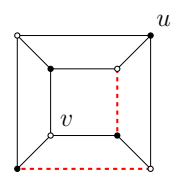

(f)

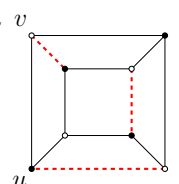

(g)

Fig. 1: The minimal bad configurations in $Q_{3}$.

Proof: First observe that each configuration $(u, v, M)$ in Figure 1 is bad. Note that $M$ is presented with dashed lines. Moreover, observe that there are no two configurations in Figure 1 such that the first one contains the other one.

Now we show that the configuration $(u, v, M)$ is either good or contains one of the bad configurations in Figure 1 1 . The hypercube $Q_{3}$ has 10 non-isomorphic matchings, including the empty one, depicted in Figure 2 (1)-(10). Note that in this figure the edges of the matchings are presented with dashed lines. So $M$ is one of the following cases in Figure 2.

(1), (2): In these cases $M$ has at most one edge, thus the configuration $(u, v, M)$ is good by Lemma 2

(3), (6): Observe that if $u \in\left\{u_{1}, u_{2}\right\}$ and $v \in\left\{v_{1}, v_{2}\right\}$, then the configuration $(u, v, M)$ is good. Otherwise it contains a configuration of type (a), (b), or (c) in Figure 1

(4): Observe that the configuration $(u, v, M)$ is good unless it equals $\left(u_{1}, v_{1}, M\right)$ or $\left(u_{2}, v_{2}, M\right)$ which are of type (d) in Figure 1.

(5): Observe that the configuration $(u, v, M)$ is good unless it equals $\left(u_{1}, v_{1}, M\right),\left(u_{1}, v_{2}, M\right)$, or $\left(u_{2}, v_{2}, M\right)$ which are of type (e) or (f) in Figure 1.

(7): In this case $Q_{3}-M-x y$ is a Hamiltonian cycle. Thus the configuration $(u, v, M)$ is good if $u v$ is an edge on this cycle. Furthermore, if the configuration $(u, v, M)$ equals $\left(u_{1}, v_{1}, M\right)$ or $\left(u_{2}, v_{2}, M\right)$, observe that it is also good. Otherwise it contains a configuration of type (e) or (f) in Figure 1

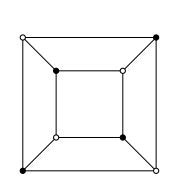

(1)

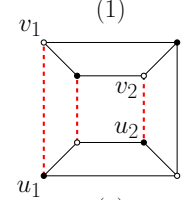

(6)
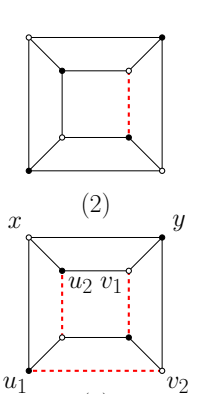

(7)

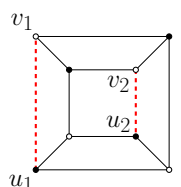

(3)

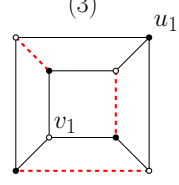

(8)

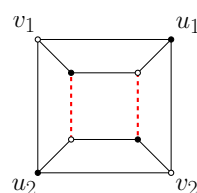

(4)

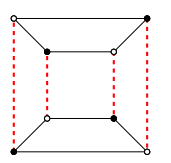

(9)

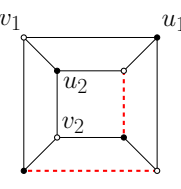

(5)

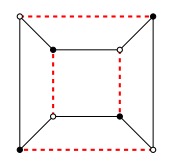

(10)

Fig. 2: All matchings in $Q_{3}$ up to isomorphism. 
(8): Observe that if $u v \in M$, then the configuration $(u, v, M)$ is good. Furthermore, it is also good if $d(u, v)=3$ and $\{u, v\} \neq\left\{u_{1}, v_{1}\right\}$. Otherwise it contains a configuration of type (e), (f), or (g) in Figure1.

(9): In this case $Q_{3}-M$ is disconnected so $(u, v, M)$ is a bad configuration. Observe that it contains a configuration of type (a), (b), or (c) in Figure 1

(10): In the last case $Q_{3}-M$ is a Hamiltonian cycle. Thus the configuration $(u, v, M)$ is good if $u v$ is an edge on this cycle. Otherwise it contains a configuration of type (e) or (f) in Figure 1.

This completes the analysis of all cases and establishes the lemma.

The following two lemmas will be useful for inquiry of $Q_{4}$ in the next section. The first lemma applies typically for configurations $(u, v, M)$ such that both $u$ and $v$ belong to a same subcube $Q_{L}^{d}$ or $Q_{R}^{d}$ which contains at most 2 edges of $M$.

Lemma 10 Let $u$ and $v$ be vertices of different parity and let $e_{1}$ and $e_{2}$ be distinct edges in $Q_{3}$. Then there is a Hamiltonian path of $Q_{3}$ between $u$ and $v$ that

(i) contains exactly one of $e_{1}$ and $e_{2}$; or

(ii) avoids both $e_{1}, e_{2}$ but contains an edge $x y$ such that $x \in e_{1}$ and $y \in e_{2}$.

Proof: Suppose first that $u$ and $v$ are at distance 3. From Figure 3.1) it is obvious that all Hamiltonian paths between $u$ and $v$ are isomorphic. Moreover, each of them contains one edge of the upper level, one edge of the lower level, and five edges of the middle level. Considering few cases, regarding the levels that contain $e_{1}$ and $e_{2}$, it is easy to find a suitable path.

Suppose now that $u v$ is an edge of $Q_{3}$. We distinguish three cases:

Case 1: Edges $e_{1}$ and $e_{2}$ are adjacent at some vertex $x$. If $x=u$, then assume that $e_{1}=u y \neq u v$ and apply Lemma 1 to find a Hamiltonian path of $Q_{3}-u$ between $y$ and $v$. Extending this path with the edge $e_{1}=u y$ we obtain a Hamiltonian path of $Q_{3}$ between $u$ and $v$ that contains $e_{1}$ and avoids $e_{2}$. If $x=v$, then proceed similarly. Otherwise we have $x \notin\{u, v\}$. The Hamiltonian path between $u$ and $v$ that avoids $e_{1}$ from Lemma 2 contains $e_{2}$ since it passes through the vertex $x$.

Case 2: Edges $e_{1}$ and $e_{2}$ are at distance 1. If the edge $u v$ is adjacent to both $e_{1}$ and $e_{2}$, then we have two non-isomorphic cases in Figures 3 (2)-(3) with desired Hamiltonian paths. Otherwise, we may assume that $x \in e_{1}, y \in e_{2}$, and $y \notin\{u, v\}$ for some edge $x y$. The Hamiltonian path between $u$ and $v$ that avoids $e_{1}$ from Lemma 2 contains $e_{2}$ or $x y$ since it passes through the vertex $y$.

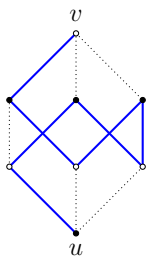

(1)

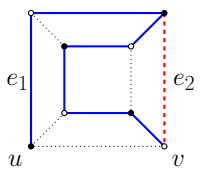

(2)

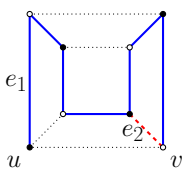

(3)

Fig. 3: An illustration for Lemma 10 


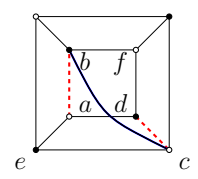

Fig. 4: The configuration of Lemma11
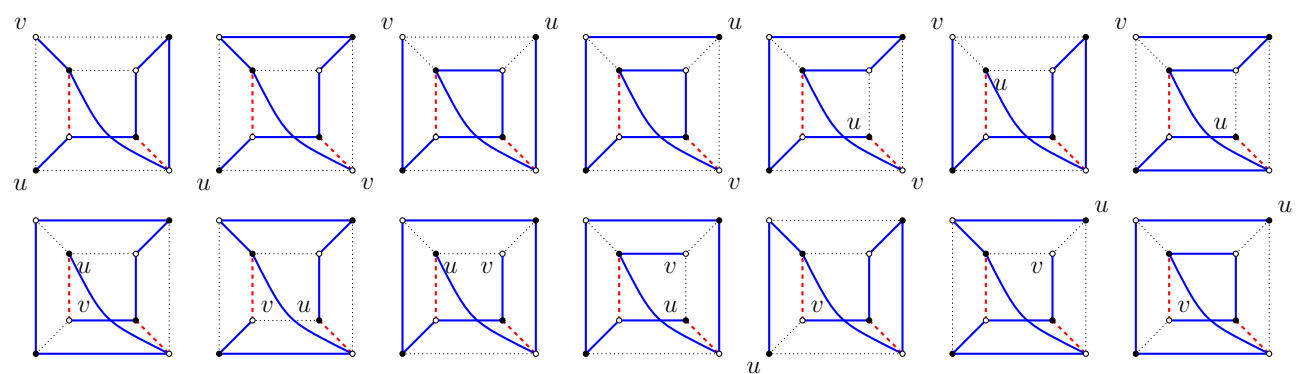

Fig. 5: Hamiltonian paths in Lemma 11 for each pair $\{u, v\}$ of endvertices.

Case 3: Edges $e_{1}$ and $e_{2}$ are at distance 2. If $e_{1}=u v$, apply Corollary 4 to find a Hamiltonian path between $u$ and $v$ that contains edge $e_{2}$. If $e_{2}=u v$, proceed similarly. Otherwise the configuration $\left(u, v,\left\{e_{1}, e_{2}\right\}\right)$ is bad since it is of type (a), (b), or (c) in Figure 1 Thus the Hamiltonian path between $u$ and $v$ that avoids $e_{1}$ from Lemma 2 contains $e_{2}$. This completes the analysis of all cases.

The second lemma applies for example when $M_{R}^{d}=\{a b, c d\}$ as in Figure 4 and $Q_{L}^{d}$ contains a path between vertices $b^{d}$ and $c^{d}$. The extra edge $b c$ represents this path.

Lemma 11 Let $a, b, c, d, e, f$ be vertices of $Q_{3}$ as depicted in Figure 4 and $u, v \in V\left(Q_{3}\right)$ be any two vertices of different parity. Then $Q_{3}+b c$ has a Hamiltonian path between $u$ and $v$ that contains the edge bc and avoids $\{a b, c d\}$ unless $\{u, v\}=\{b, c\}$ or $\{u, v\}=\{e, f\}$.

Proof: By exhausting all 14 possibilities for $\{u, v\}$, as it is presented in Figure 5 , one can verify the validity of the lemma.

\section{Appendix B: Gray codes in $Q_{4}$}

If $M$ is aligned in $Q_{4}$, we can use Lemma 7 which holds for every $n \geq 4$. Thus, it remains to consider admissible configurations $(u, v, M)$ only with an unaligned matching $M$. We start with the list of all maximal unaligned matchings in $Q_{4}$.

Let $m(M)$ denote the maximum number of edges of $M$ of the same dimension, i. e., $m(M)=$ $\max _{d \in[n]}\left|M^{d}\right|$.

Lemma 12 Let $M$ be a maximal unaligned matching in $Q_{4}$. Then $m(M) \in\{2,3,4\}$ and $M$ is isomorphic to one of the matchings in Figures 68.8.

Proof: Let $d \in[4]$ be a dimension with the maximal number of edges of $M$, i. e., $\left|M^{d}\right|=m(M)$. Since $M$ is unaligned, we have $\left|M_{L}^{d}\right| \geq 2$ or $\left|M_{R}^{d}\right| \geq 2$, say $\left|M_{L}^{d}\right| \geq 2$. Thus among eight vertices of 


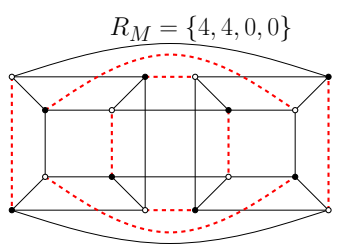

(1)

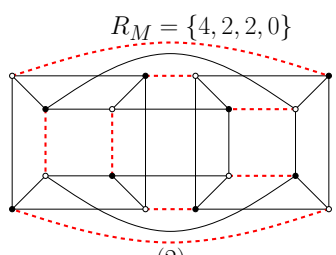

2)

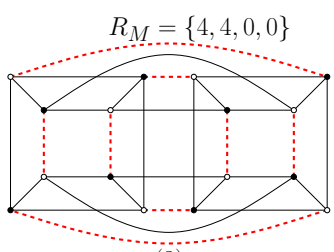

(3)

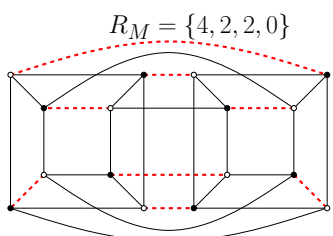

(4)

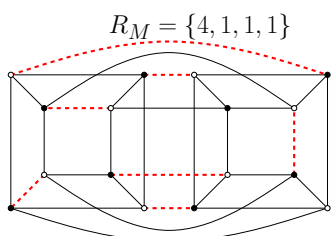

(5)

Fig. 6: All maximal unaligned matchings $M$ of $Q_{4}$ with $m(M)=4$.

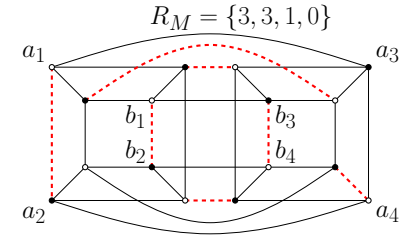

(1)

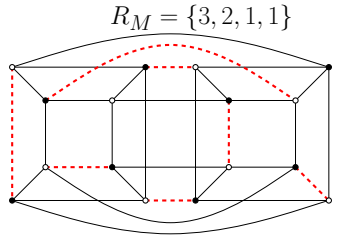

(2)

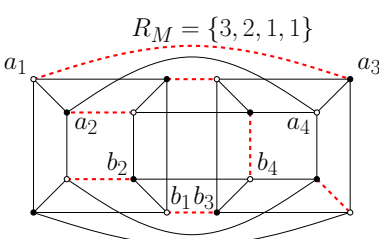

(4)

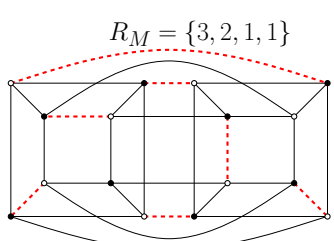

(5)

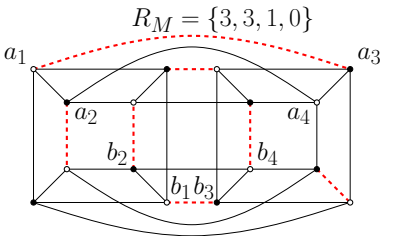

(3)

Fig. 7: All maximal unaligned matchings $M$ of $Q_{4}$ with $m(M)=3$.

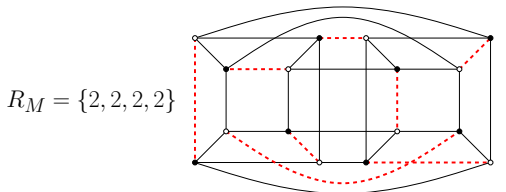

(1)

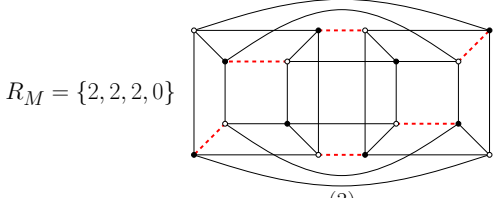

(3)

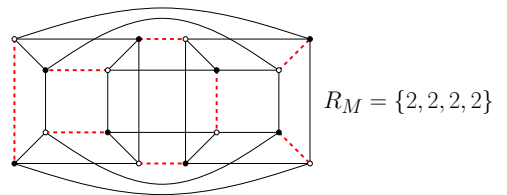

(2)

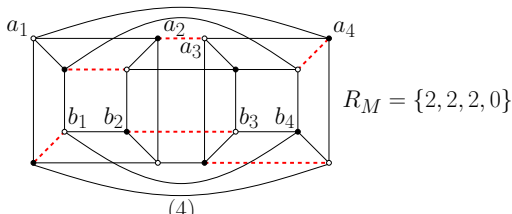

(4)

Fig. 8: All maximal unaligned matchings $M$ of $Q_{4}$ with $m(M)=2$. 


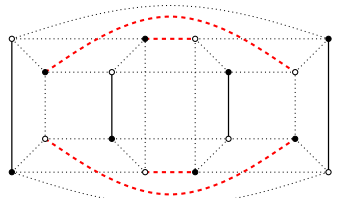

(1)

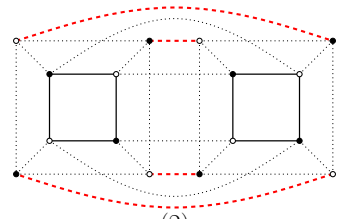

(2)

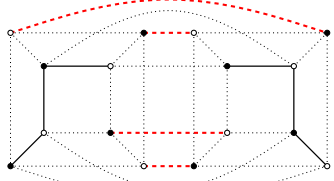

(3)

Fig. 9: All sets $M^{d}$ with 4 edges in a maximal unaligned matching $M$ of $Q_{4}$.

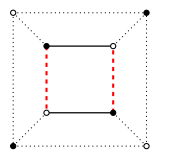

(1)

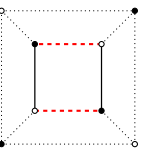

(2)

Fig. 10: All maximal matchings of $G$ in Subcase 1.2.

$Q_{L}^{d}$ there are at most four vertices that are not matched by $M_{L}^{d}$. Hence $m(M) \leq 4$. On the other hand, every maximal matching in $Q_{4}$ contains at least 6 edges [6], hence at least 2 edges from $M$ are of a same dimension. This implies that $m(M) \geq 2$. So $m(M) \in\{2,3,4\}$.

Let $G_{L}$ and $G_{R}$ be the graphs obtained from $Q_{L}^{d}$ and $Q_{R}^{d}$, respectively, by removing all vertices incident with $M^{d}$. Note that $G_{L}$ and $G_{R}$ are isomorphic, so let us write simply $G$ when it is clear from the context for which of the graphs we speak. Since $M$ is maximal, both $M_{L}^{d}$ and $M_{R}^{d}$ are maximal matchings of $G$.

To show that $M$ is one of the matchings in Figures 6, we first consider the set $M^{d}$ and then the combination of sets $M_{L}^{d}$ and $M_{R}^{d}$. The matchings $M^{d}, M_{L}^{d}$ and $M_{R}^{d}$ in the following figures are represented by dashed lines, the edges of the graph $G$ are bold, and the remaining edges are dotted.

Note that in the following figures the isomorphic graphs $G_{L}$ and $G_{R}$ are presented in a mirrored way, so the matchings $M_{R}^{d}$ and the maximal matchings of $G$ are also presented in a mirrored way.

Case 1: $m(M)=4$. Since $M$ is unaligned, there are three non-isomorphic combinations of four edges in $M^{d}$; see Figure 9. We consider now three possibilities.

Subcase 1.1: $M^{d}$ is as in Figure 9 1). Obviously, there is only one maximal matching of $G$. Thus $M_{L}^{d}$ and $M_{R}^{d}$ are uniquely determined and the matching $M$ is as in Figure 6.(1).

Subcase 1.2: $M^{d}$ is as in Figure 9 2). There are two maximal matchings of $G$ depicted in Figure 10 By symmetry, we may assume that $M_{L}^{d}$ is as in Figure 10.1 . Now, if $M_{R}^{d}$ is also as in Figure 10.1), the matching $M$ corresponds to Figure 6.(3). And, if $M_{R}^{a}$ is as in Figure 10.2), then $M$ corresponds to Figure 6(2).

Subcase 1.3: $M^{d}$ is as in Figure 9 3). There are two maximal matchings of $G$ depicted in Figure 12. By the maximality of $M$, it follows that $M_{L}^{d}$ and $M_{R}^{d}$ are not both as in Figure 12(2). Now, if both of them are as in Figure 12(1), we infer Figure 6(4). Finally, if one of them is as in Figure 12(1), and the other one as in Figure 12(2), we infer Figure 6(5).

Case 2: $m(M)=3$. Since $M$ is unaligned, there are two non-isomorphic combinations of three edges in $M^{d}$ which are depicted in Figure 11 Consider now these two cases separately.

Subcase 2.1: $M^{d}$ is as in Figure 11 1). There are three maximal matchings of $G$ depicted in Figure 13 By symmetry, we may assume that if $M_{L}^{d}$ is as in Figure $13(x)$, then $M_{R}^{d}$ is as in Figure $13(y)$ where $1 \leq x \leq y \leq 3$. Moreover, since $M$ is a maximal matching, we infer $x \neq y$. We have the following 


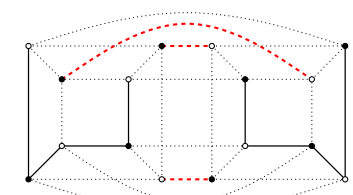

(1)

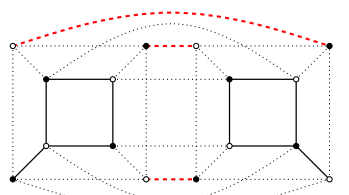

(2)

Fig. 11: All sets $M^{d}$ with 3 edges in a maximal unaligned matching $M$ of $Q_{4}$.

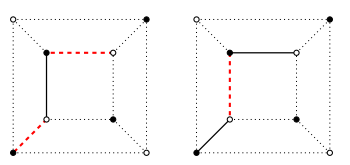

(1)

(2)

Fig. 12: All maximal matchings of $G$ in Subcase 1.3.

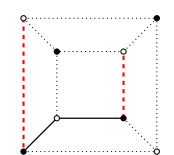

(1)

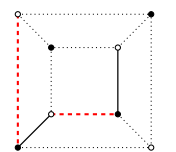

(2)

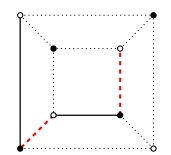

(3)

Fig. 13: All maximal matchings of $G$ in Subcase 2.1.

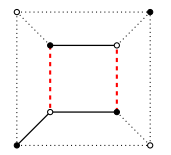

(1)

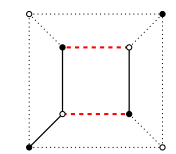

(2)

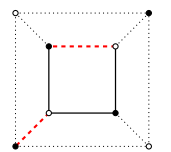

(3)

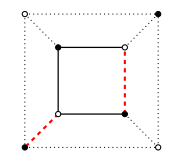

(4)

Fig. 14: All maximal matchings of $G$ in Subcase 2.2. 
possibilities for the pair $(x, y)$ :

$(1,3)$ : The matching $M$ is as in Figure $7(1)$;

$(2,3)$ : The matching $M$ is as in Figure $7(2)$;

$(1,2)$ : This case is isomorphic to the case $(1,3)$. Indeed, consider the isomorphism of $Q_{4}$ that interchanges vertices $a_{i}$ and $b_{i}$ in Figure $7(1)$ for every $i \in[4]$ and fixes all other vertices.

Subcase 2.2: $M^{d}$ is as in Figure 11 2). There are four maximal matchings of $G$ depicted in Figure 14 By symmetry, we may assume that if $M_{L}^{d}$ is as in Figure $14(x)$, then $M_{R}^{d}$ is as in Figure $14(y)$ where $1 \leq x \leq y \leq 4$. Moreover, since $M$ is a maximal matching, it follows that $x \neq y$. Thus, we have the following possibilities for the pair $(x, y)$ :

$(1,4)$ : The matching $M$ is as in Figure $7(3)$;

$(2,4)$ : The matching $M$ is as in Figure $7(4)$;

$(3,4)$ : The matching $M$ is as in Figure $7(5)$;

$(1,2)$ : This case does not occur since $M$ is a maximal matching;

$(1,3)$ and $(2,3)$ : These cases are isomorphic to the cases $(2,4)$ and $(1,4)$, respectively. Indeed, consider the isomorphism of $Q_{4}$ that interchanges vertices $a_{i}$ and $b_{i}$ in Figures 74 ) and 773) for every $i \in[4]$ and that fixes all other vertices.

Case 3: $m(M)=2$. Let $k(M)$ be the minimal distance of distinct edges of $M$ of a same dimension, i. e.,

$$
k(M)=\min \left\{d\left(e_{1}, e_{2}\right) \mid e_{1}, e_{2} \in M^{i} \text { for some } i \in[4] \text { and } e_{1} \neq e_{2}\right\} .
$$

We assume that $M^{d}$ contains edges at distance $k(M)$, otherwise choose $d$ accordingly. For each $k(M) \in$ [3] we have $M^{d}$ in Figure 15

Subcase 3.1: $k(M)=3$. Thus $M^{d}$ is as in Figure 15(1). There are five maximal matchings of $G$ depicted in Figure 16 Observe that neither $M_{L}^{d}$ nor $M_{R}^{d}$ is as in Figures 16(3)-(5) since $k(M)=3$. Moreover, $M_{d}^{L}$ and $M_{d}^{R}$ are not both as in Figure 16.(1), or both as in Figure 16.2) since $k(M)=3$. Thus, one of them is as in Figure 16,1), and the other one as in Figure 16(2). Hence $M$ corresponds to Figure $8(1)$.

Subcase 3.2: $k(M)=2$. Thus $M^{d}$ is as in Figure 15(2). There are seven maximal matchings of $G$ depicted in Figure 17 Observe that neither $M_{L}^{d}$ nor $M_{R}^{d}$ is as in Figures 17(6)-(7) since $k(M)=2$. Furthermore, neither $M_{L}^{d}$ nor $M_{R}^{d}$ is as in Figure 17,5) since all matchings of $G$ in Figures 17(1)-(5) contain at least one edge of $e_{1}$ or $e_{2}$ and $k(M)=2$. By symmetry, we may assume that if $M_{L}^{d}$ is as in Figure $17(x)$, then $M_{R}^{d}$ is as in Figure $17(y)$ where $1 \leq x \leq y \leq 4$. Moreover, $x \neq y$ since $M$ is a maximal matching. We have the following possibilities for the pair $(x, y)$ :

$(1,2)$ : The matching $M$ is as in Figure $8(4)$;

$(3,4)$ : This case is isomorphic to the case $(1,2)$. Indeed, consider the isomorphism of $Q_{4}$ that interchanges vertices $a_{i}$ and $b_{i}$ in Figure 8(4) for every $i \in[4]$ and fixes all other vertices.

$(1,3),(1,4),(2,3)$, and $(2,4)$ : These cases do not occur since $M$ is a maximal matching. 


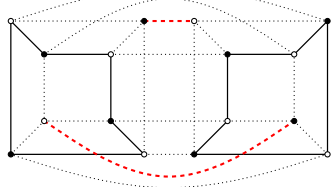

(1)

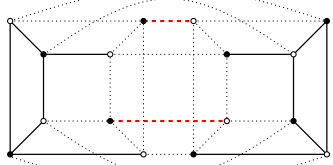

(2)

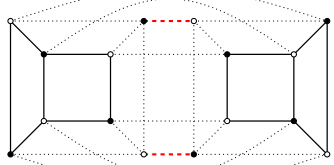

(3)

Fig. 15: All sets $M^{d}$ with 2 edges in a maximal unaligned matching $M$ of $Q_{4}$.

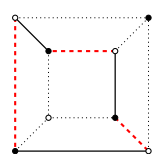

(1)

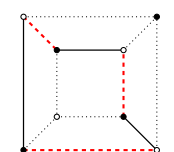

(2)

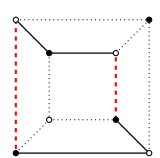

(3)

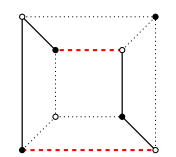

(4)

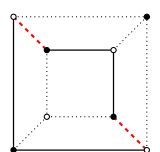

(5)

Fig. 16: All maximal matchings of $G$ in Subcase 3.1.

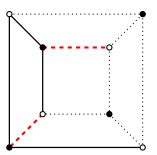

(1)

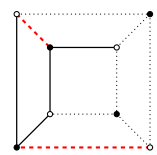

(2)

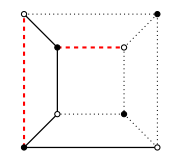

(3)

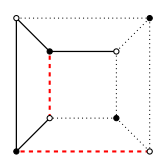

(4)

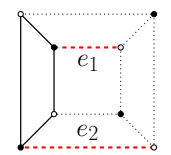

(5)

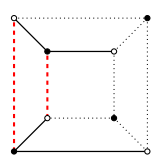

(6)

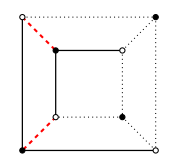

(7)

Fig. 17: All maximal matchings of $G$ in Subcase 3.2.

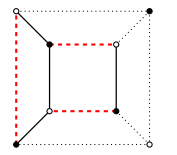

(1)

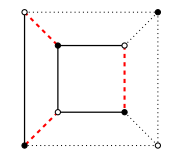

(2)

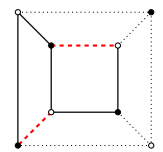

(3)

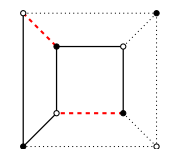

(4)

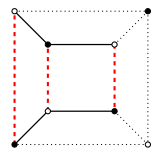

(5)

Fig. 18: All maximal matchings of $G$ in Subcase 3.3. 
Subcase 3.3: $k(M)=1$. Thus $M^{d}$ is as in Figure 15.(3). There are five maximal matchings of $G$ depicted in Figure 18 Observe that neither $M_{L}^{d}$ nor $M_{B}^{d}$ is as in Figure 18.5) since $m(M)=2$. By symmetry, we may assume that if $M_{L}^{d}$ is as in Figure $18(x)$, then $M_{R}^{d}$ is as in Figure $18(y)$ where $1 \leq x \leq y \leq 4$. We have the following possibilities for the pair $(x, y)$ :

$(1,2)$ : The matching $M$ is as in Figure $8(2)$;

$(3,4)$ : The matching $M$ is as in Figure $8(3)$;

$(1,1),(1,3),(1,4),(2,2),(2,3)$, and $(2,4)$ : These cases do not occur since $m(M)=2$;

$(3,3)$ and $(4,4)$ : These cases do not occur since $M$ is a maximal matching.

Note that the choice of the dimension $d$ was not determined uniquely. Therefore, to conclude the proof, we need to verify that the matchings in Figures 6 are non-isomorphic.

Define the mark of $M$ to be the collection $R_{M}=\left\{\left|M^{d}\right|\right\}_{d \in[n]}$. Clearly, matchings with different marks are not isomorphic. So we need to examine only matchings with different marks in Figures 6, 8 . This can be easily verified by the reader.

Lemma 13 Let $(u, v, M)$ be a maximal admissible configuration in $Q_{n}$ with an unaligned matching $M$. Then $M$ is maximal with respect to inclusion.

Proof: Suppose on the contrary that $M$ is not maximal, i. e., $M \cup\{e\}$ is a matching for some edge $e \in E\left(Q_{n}\right) \backslash M$. Let $d$ be the dimension of $e$. Since $M$ is unaligned, we have $\left|M_{L}^{d}\right| \geq 2$ or $\left|M_{R}^{d}\right| \geq 2$, so we may assume that $M_{L}^{d}$ contains edges $x_{1} x_{2}$ and $x_{3} x_{4}$. Let $e_{i}=x_{i} x_{i}^{d}$ for $i \in$ [4].

Since $d\left(e_{1}, e_{2}\right)$ is odd, $d\left(e_{3}, e_{4}\right)$ is odd, $e_{1}, e_{2}, e_{3}, e_{4}$ are not in $M \cup\{e\}$, and $(u, v, M)$ is admissible, it follows from the definition that $(u, v, M \cup\{e\})$ is also admissible. But this contradicts the assumption that $(u, v, M)$ is a maximal admissible configuration.

Now we are ready to find a Hamiltonian path of $Q_{4}-M$ between $u$ and $v$ if $(u, v, M)$ is admissible configuration. The following lemma serves as a basis of induction in the next section.

Lemma 14 Let $M$ be a matching in $Q_{4}$ and $u, v \in V\left(Q_{4}\right)$. If $(u, v, M)$ is admissible, then there is a Hamiltonian path of $Q_{4}-M$ between $u$ and $v$.

Proof: If $M$ is aligned, we can use Lemma 7 which holds for every $n \geq 4$. Thus, it remains to consider admissible configurations $(u, v, M)$ only with unaligned matching $M$.

Assume that $(u, v, M)$ is a maximal admissible configuration, otherwise introduce new edges in $M$. Notice that with this procedure $M$ stays unaligned. By Lemma 13 , the matching $M$ is maximal. Thus $m(M) \in\{2,3,4\}$ and $M$ corresponds to one of the matchings in Figures 6 by Lemma 12 .

Let $d \in[4]$ be a dimension with the maximal number of edges from $M$, i. e., $\left|M^{d}\right|=m(M)$. Now, as $d$ is fixed, for the sake of simplicity, let us omit $d$ in the notions of $Q_{L}^{d}, Q_{R}^{d}, M_{L}^{d}$, and $M_{R}^{d}$. Furthermore, for $x \in V\left(Q_{R}\right)$ and $y \in V\left(Q_{L}\right)$ let $x_{L}$ and $y_{R}$ denote their corresponding vertices in the opposite subcube, i. e., $x_{L}=x^{d}$ and $y_{R}=y^{d}$. We say that vertices $u$ and $v$ are separated if $u \in V\left(Q_{L}\right)$ and $v \in V\left(Q_{R}\right)$ or vice versa. 
In what follows, we distinguish three cases regarding $m(M)$. In each case we consider good/bad configurations in $Q_{L}$ and $Q_{R}$. Then, regarding the position of vertices $u$ and $v$, we look separately in $Q_{L}$ and $Q_{R}$ for paths that can be combined into a Hamiltonian path of $Q_{4}-M$ between $u$ and $v$.

Case 1: $m(M)=4$. Applying Lemma 9, observe that Figure 20(1)-(5) shows all bad configurations in $Q_{L}$ or $Q_{R}$ for each of the five maximal unaligned matchings $M$ with $m(M)=4$ depicted in Figure 6 , respectively. Vertices $u$ and $v$ that form a bad configuration $\left(u, v, M_{L}\right)$ in $Q_{L}$ or $\left(u, v, M_{R}\right)$ in $Q_{R}$ are encompassed by a solid elliptic curve, the matching $M$ is represented by dashed edges, whereas other edges of $Q_{4}$ are omitted for the sake of clarity. For example, in Figure 20(2) we have two bad configurations in $Q_{L}$ and two bad configurations in $Q_{R}$.

Subcase 1.1: Vertices $u$ and $v$ are separated. Assume that $u \in V\left(Q_{L}\right)$ and $v \in V\left(Q_{R}\right)$. We distinguish the following two possibilities.

1.1.1: $M$ is as in Figure 20 (1) and $u$ or $v$ is incident with $M^{d}$. By symmetry, we may assume that $u=a$. Observe in Figure 21 that the statement of the lemma holds for each $v \in V\left(Q_{R}\right)$ of different parity than $u$. The Hamiltonian paths are presented by bold edges.

1.1.2: $M$ is as in Figure 20(2)-(5) or none of $u$ and $v$ is incident with $M^{d}$. Let $w \in V\left(Q_{L}\right)$ be a vertex of different parity than $u$ such that it is incident with $M_{L}$. Then, $v$ and $w^{R}$ are also of different parities. Observe in Figure 20.2)-(5) that the vertices $w$ and $w^{R}$ are in no bad configuration in $Q_{L}$ and $Q_{R}$, respectively. Thus the configurations $\left(u, w, M_{L}\right)$ and $\left(v, w_{R}, M_{R}\right)$ are good.

Hence there is a Hamiltonian path $P_{u w}$ of $Q_{L}-M_{L}$, and a Hamiltonian path $P_{w_{R} v}$ of $Q_{R}-M_{R}$. The desired Hamiltonian path of $Q_{4}-M$ is $P_{u w}+P_{w_{R} v}$.

Subcase 1.2: Both vertices $u$ and $v$ are in $Q_{L}$. Note that $M_{L}$ is of size 2 , thus denote by $e_{1}, e_{2}$ these two edges. By Lemma 10, there is a Hamiltonian path $P_{u v}$ that either contains precisely one of $e_{1}$ and $e_{2}$, or avoids both $e_{1}, e_{2}$ but contains an edge adjacent to both $e_{1}, e_{2}$. Let $x y \in E\left(Q_{L}\right)$ be that contained edge. Observe in Figure 20(1)-(5) that there is no bad configuration $\left(w, z, M_{R}\right)$ in $Q_{R}$ such that $w_{L}$ and $z_{L}$ are both incident with $e_{1}$ or $e_{2}$. Thus the configuration $\left(x_{R}, y_{R}, M_{R}\right)$ is good.

Hence there is a Hamiltonian path $P_{x_{R} y_{R}}$ of $Q_{R}-M_{R}$. The desired Hamiltonian path of $Q_{4}-M$ is $P_{u x}+P_{x_{R} y_{R}}+P_{y v}$ where $P_{u x}$ and $P_{y v}$ are disjoint subpaths of $P_{u v}$, assuming that $x$ is closer to $u$ on $P_{u v}$ than $y$.

Subcase 1.3: Both vertices $u$ and $v$ are in $Q_{R}$. If $M$ is as in Figure 20(1)-(4), we have symmetry to the previous subcase. However, if $M$ is as in Figure 20(5), then $M_{R}$ contains only one edge $e_{1}$. Then just put $e_{2}=b c$ and proceed symmetrically as in Subcase 1.2.

Case 2: $m(M)=3$. Applying Lemma 9 observe that Figure 19.(1)-(5) shows all bad configurations in $Q_{L}$ or $Q_{R}$ for each of the five maximal unaligned matchings $M$ with $m(M)=3$ depicted in Figure 7 . respectively. We consider the following possibilities.

Subcase 2.1: Vertices $u$ and $v$ are separated. Again, assume that $u \in V\left(Q_{L}\right)$ and $v \in V\left(Q_{R}\right)$. We distinguish the following two possibilities.

2.1.1: $M$ is as in Figure 19 1) and $u$ is not incident with $M_{L}$. Observe in Figure 22 that for each $u \in V\left(Q_{L}\right)$ that is not incident with $M_{L}$, there are spanning paths $P_{u w_{L}}$ and $P_{b_{L} c_{L}}$ of $Q_{4}-M_{L}$. By Lemma 11, there is a Hamiltonian path $P_{w v}$ of $Q_{R}+b c$ that contains the edge $b c$ and avoids $M_{R}$.

Hence a desired Hamiltonian path of $Q_{4}-M$ is $P_{u w_{L}}+P_{w b}+P_{b_{L} c_{L}}+P_{c v}$ where $P_{w b}$ and $P_{c v}$ are disjoint subpaths of $P_{w v}$, assuming that $b$ is closer to $w$ on $P_{w v}$ than $c$.

2.1.2: $M$ is as in Figure 19 2)-(5) or $u$ is incident with $M_{L}$. Let $x, y \in V\left(Q_{L}\right)$ be vertices of different parity than $u$ that are incident with $M_{L}$. We can always choose such $x, y$ since $\left|M_{L}\right|=2$. Observe in Figure 19.(1) that each bad configuration in $Q_{L}$ includes at most one vertex incident with $M_{L}$. Similarly, 


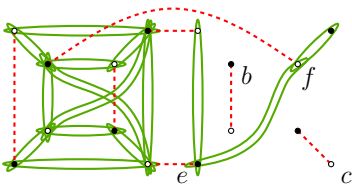

(1)

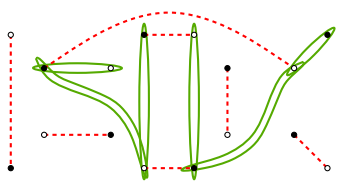

$(2)$

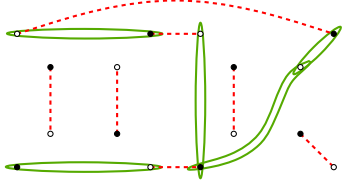

(3)

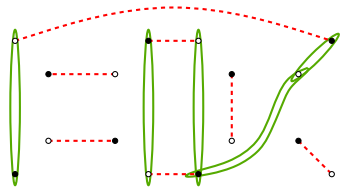

(4)

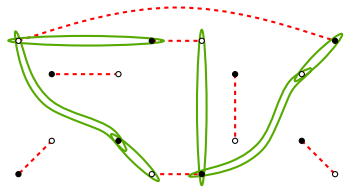

(5)

Fig. 19: All bad configurations in $Q_{L}$ or $Q_{R}$ for each maximal unaligned matching $M$ in $Q_{4}$ with $m(M)=3$.

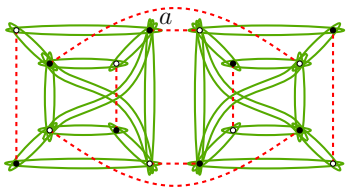

(1)

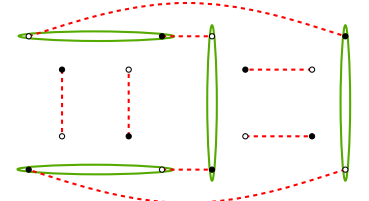

$(2)$

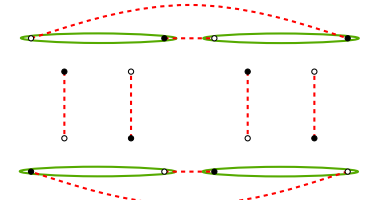

(3)

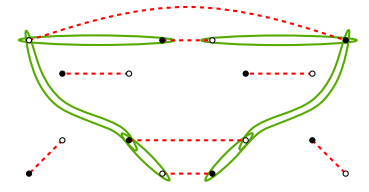

(4)

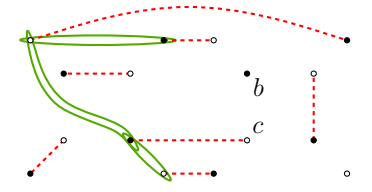

(5)

Fig. 20: All bad configurations in $Q_{L}$ or $Q_{R}$ for each maximal unaligned matching $M$ in $Q_{4}$ with $m(M)=4$.
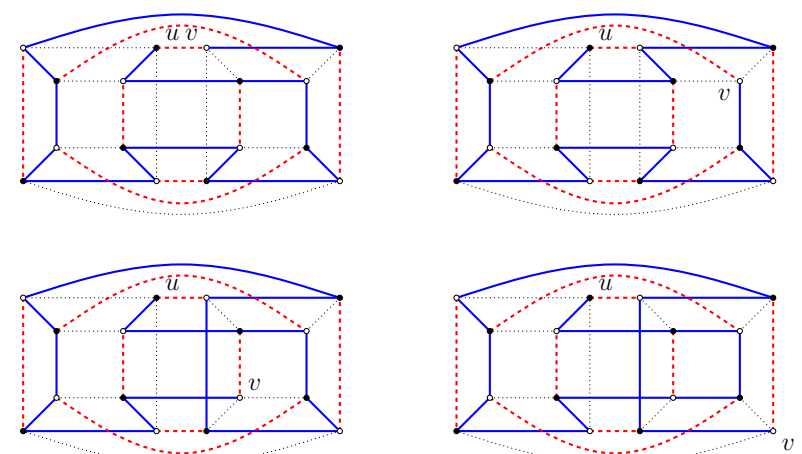

Fig. 21: Hamiltonian paths for Subcase 1.1.1. 
observe in Figure 19.2)-(5) that each bad configuration in $Q_{L}$ includes no vertex incident with $M_{L}$. Thus both configurations $\left(u, x, M_{L}\right)$ and $\left(u, y, M_{L}\right)$ are good. Moreover, observe in Figure 19.1)-(5) that for at most one $z \in V\left(Q_{L}\right)$, if $z$ is incident with $M_{L}$, then $z_{R}$ is in a bad configuration in $Q_{R}$. Hence at most one of configurations $\left(v, x_{R}, M_{R}\right)$ and $\left(v, y_{R}, M_{R}\right)$ is bad. Let $w \in\{x, y\}$ be such that the configuration $\left(v, w_{R}, M_{R}\right)$ is good.

Therefore, there is a Hamiltonian path $P_{u w}$ of $Q_{L}-M_{L}$, and a Hamiltonian path $P_{w_{R} v}$ of $Q_{R}-M_{R}$. Finally, the desired Hamiltonian path is $P_{u w}+P_{w_{R} v}$.

Subcase 2.2: Vertices $u$ and $v$ are both in $Q_{L}$ or both in $Q_{R}$. We distinguish the following two possibilities.

2.2.1: $M$ is as in Figure 19 1 ) and both $u$ and $v$ are in $Q_{R}$. If $\{u, v\}=\{b, c\}$ or $\{u, v\}=\{e, f\}$, observe in Figure 23 that the statement of the lemma holds. Otherwise, by Lemma 11, there is a Hamiltonian path $P_{u v}$ of $Q_{R}+b c$ that contains the edge $b c$ and avoids $M_{R}$. Observe in Figure 19.1) that the configuration $\left(b_{L}, c_{L}, M_{L}\right)$ is good.

Thus there is a Hamiltonian path $P_{b_{L} c_{L}}$ of $Q_{L}-M_{L}$. The desired Hamiltonian path of $Q_{4}-M$ is $P_{u b}+P_{b_{L} c_{L}}+P_{c v}$ where $P_{u b}$ and $P_{c v}$ are disjoint subpaths of $P_{u v}$, assuming that $b$ is closer to $u$ on $P_{u v}$ than $c$.

2.2.2: $M$ is as in Figure 19 1) and both $u$ and $v$ are in $Q_{L}$, or $M$ is as in Figure 19 2)-(5). We proceed similarly as in Subcase 1.2. Assume that $u, v \in V\left(Q_{L}\right)$, otherwise interchange the roles of $Q_{L}$ and $Q_{R}$. Let $M_{L}=\left\{e_{1}, e_{2}\right\}$. By Lemma 10, there is a Hamiltonian path $P_{u v}$ that either contains precisely one of $e_{1}$ and $e_{2}$, or avoids both $e_{1}, e_{2}$ but contains an edge adjacent to both $e_{1}, e_{2}$. Let $x y$ be that contained edge. Observe in Figure 19.1)-(5) that there is no bad configuration $\left(w, z, M_{R}\right)$ in $Q_{R}$ such that $w_{L}$ and $z_{L}$ are both incident with $e_{1}$ or $e_{2}$. Thus the configuration $\left(x_{R}, y_{R}, M_{R}\right)$ is good.

Hence there is a Hamiltonian path $P_{x_{R} y_{R}}$ of $Q_{R}-M_{R}$. The desired Hamiltonian path of $Q_{4}-M$ is $P_{u x}+P_{x_{R} y_{R}}+P_{y v}$ where $P_{u x}$ and $P_{y v}$ are disjoint subpaths of $P_{u v}$, assuming that $x$ is closer to $u$ than $y$ on the path $P_{u v}$.

Case 3: $m(M)=2$. Applying Lemma 9 observe that Figure 24(1)-(4) shows all bad configurations in $Q_{L}$ or $Q_{R}$ for each of the four maximal unaligned matchings $M$ with $m(M)=2$ depicted in Figure 8 respectively.

Subcase 3.1: Vertices $u$ and $v$ are separated. Assume that $u \in V\left(Q_{L}\right)$ and $v \in V\left(Q_{R}\right)$. We distinguish the following three possibilities.

3.1.1: $M$ is as in Figure 24 1) and $u$ or $v$ is incident with $M^{d}$. Assume that $u=a$, the other case is symmetric. Observe in Figure 25 that the statement holds for each $v \in V\left(Q_{R}\right)$ of different parity than $u$.

3.1.2: $M$ is as in Figure 244) and $\{u, v\}=\{d, e\}$. Observe in Figure 26 that the statement holds.

3.1.3: The remaining possibilities; that is, $M$ is as in Figure 24|1) and none of $u$ and $v$ is incident with $M^{d}$, or $M$ is as in Figure 24 2)-(4) and $\{u, v\} \neq\{d, e\}$. A vertex $w \in V\left(Q_{L}\right)$ is free if it has a different parity than $u$ and is not incident with $M^{d}$.

We claim that there is a free vertex $w \in V\left(Q_{L}\right)$ such that the configurations $\left(u, w, M_{L}\right)$ and $\left(v, w_{R}, M_{R}\right)$ are good.

Suppose first that $M$ is as in Figure 24(1)-(3). Observe from the same figure that the configuration $\left(u, x, M_{L}\right)$ is bad for at most one free vertex $x$. Similarly, the configuration $\left(v, y_{R}, M_{R}\right)$ is bad for at most one free vertex $y$. Since there are three free vertices, the claim holds in this case.

Suppose now that $M$ is as in Figure 24(4). If $u$ and $d$ have different parity, then we have two free vertices and both of them satisfy the claim. If $u$ and $d$ have the same parity, we have four free vertices. 

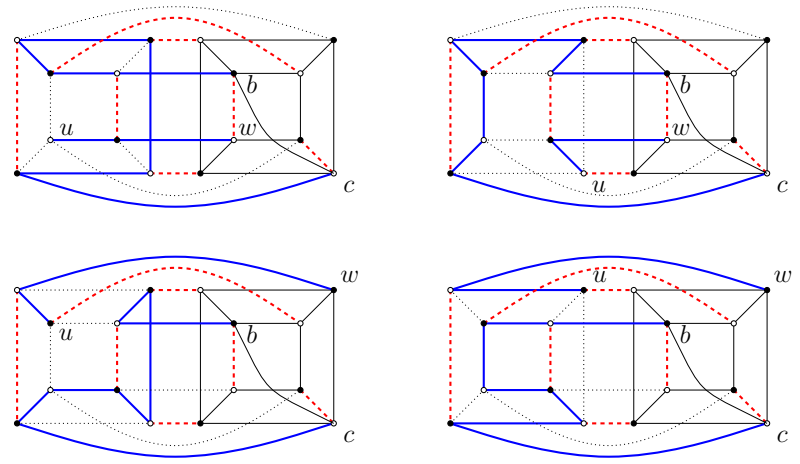

Fig. 22: Spanning paths of $Q_{L}$ for Subcase 2.1.1.
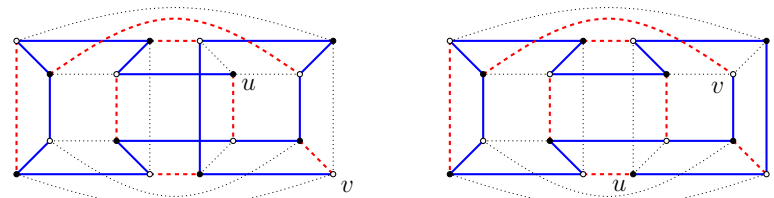

Fig. 23: Hamiltonian paths for Subcase 2.2.1.

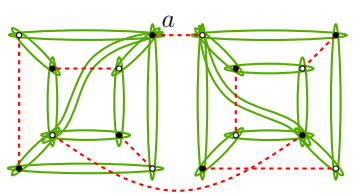

(1)

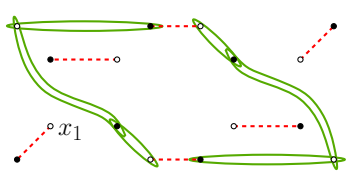

(3)

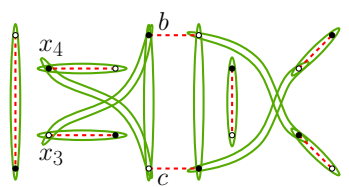

(2)

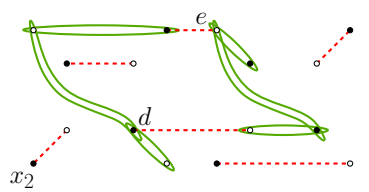

(4)

Fig. 24: All bad configurations in $Q_{L}$ or $Q_{R}$ for each maximal unaligned matching $M$ in $Q_{4}$ with $m(M)=2$. 

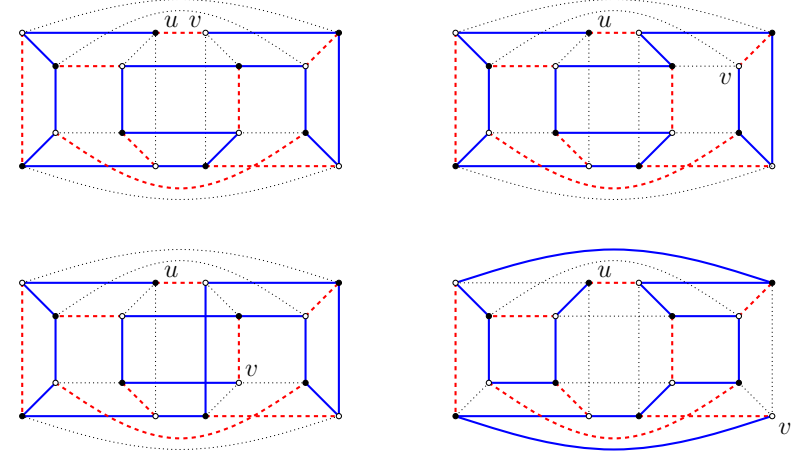

Fig. 25: Hamiltonian paths for Subcase 3.1.1.

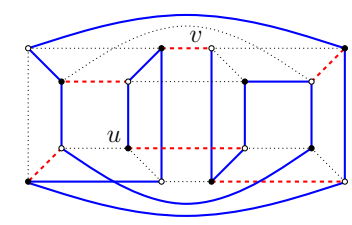

Fig. 26: A Hamiltonian path for Subcase 3.1.2.
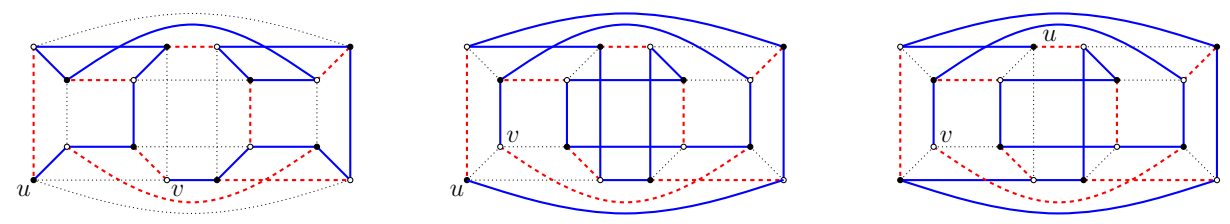

Fig. 27: Hamiltonian paths for Subcase 3.2.1.
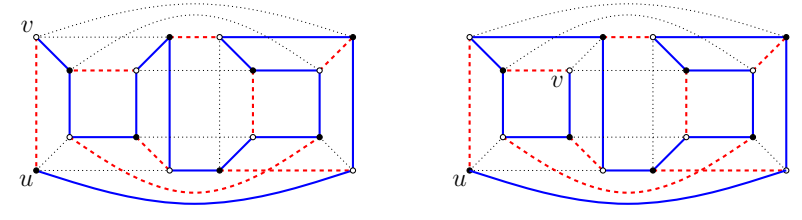

Fig. 28: Hamiltonian paths for Subcase 3.2.2.
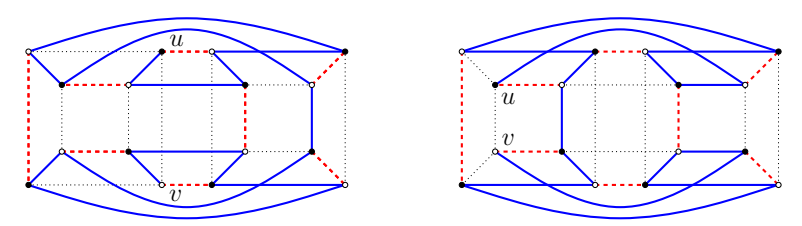

Fig. 29: Hamiltonian paths for Subcase 3.2.3. 
Observe in Figure 24(4) that the configurations $\left(u, x, M_{L}\right)$ or $\left(v, x_{R}, M_{R}\right)$ are bad for at most three free vertices $x$, since $\{u, v\} \neq\{d, e\}$. Hence the claim holds in all cases.

Therefore, there is a Hamiltonian path $P_{u w}$ of $Q_{L}-M_{L}$, and a Hamiltonian path $P_{w_{R} v}$ of $Q_{R}-M_{R}$. The desired Hamiltonian path of $Q_{4}-M$ is $P_{u w}+P_{w_{R} v}$.

Subcase 3.2: Vertices $u$ and $v$ are both in $Q_{L}$ or both in $Q_{R}$. Assume that $u, v \in V\left(Q_{L}\right)$, the case $u, v \in V\left(Q_{R}\right)$ is symmetric. We distinguish the following five possibilities.

3.2.1: $M$ is as in Figure 24 1) and the configuration $\left(u, v, M_{L}\right)$ is bad. We have three non-isomorphic cases: both, precisely one, or none of $u$ and $v$ is incident with $M_{L}$. Observe in Figure 27t that the statement holds for each of these three possibilities.

3.2.2: $M$ is as in Figure 24 1) and the configuration $\left(u, v, M_{L}\right)$ is good. We have two non-isomorphic cases: $u v \in M_{L}$ and $u v \notin M_{L}$. Observe in Figure 28 that the statement holds for both of these cases.

3.2.3: $M$ is as in Figure 24 2) and $\{u, v\}=\{b, c\}$ or $\{u, v\}=\left\{x_{3}, x_{4}\right\}$. Observe in Figure 29 that the statement holds.

3.2.4: $M$ is as in Figure 24 2)-(4), the configuration $\left(u, v, M_{L}\right)$ is good, and $\{u, v\} \neq\left\{x_{3}, x_{4}\right\}$. Then there is a Hamiltonian path $P_{u v}$ of $Q_{L}-M_{L}$.

We claim that $P_{u v}$ contains an edge $x y \in P_{u v}$ such that $x x^{R} \notin M^{d}$, yy $y^{R} \notin M^{d}$, and the configuration $\left(x_{R}, y_{R}, M_{R}\right)$ is good.

First, if $M$ is as in Figure 24(3) or 24(4), put $x=x_{1}$ or $x=x_{2}$, respectively, and choose $y$ to be any neighbor of $x$ on $P_{u v}$. Observe in Figure 24(3)-(4) that $x$ is in no bad configuration in $Q_{L}$, also $x_{R}$ is in no bad configuration in $Q_{R}, x x^{R} \notin M^{d}$, and $y y^{R} \notin M^{d}$. Thus the claim holds in this situation.

Now $M$ is as in Figure 24(2). Since $\{u, v\} \neq\left\{x_{3}, x_{4}\right\}$, we have $x_{3} \notin\{u, v\}$ or $x_{4} \notin\{u, v\}$, say $x_{3} \notin\{u, v\}$. Thus the Hamiltonian path $P_{u v}$ contains the edge $x_{3} x_{4}$. Put $x=x_{3}$ and $y=x_{4}$ and observe in Figure 24(2) that the claim is established.

Hence, by the claim, there is a Hamiltonian path $P_{x_{R} y_{R}}$ of $Q_{R}-M_{R}$. Finally, the desired Hamiltonian path of $Q_{4}-M$ is $P_{u x}+P_{x_{R} y_{R}}+P_{y v}$ where $P_{u x}$ and $P_{y v}$ are disjoint subpaths of $P_{u v}$, assuming that $x$ is closer to $u$ than $y$ on the path $P_{u v}$.

3.2.5: The remaining possibility; that is, $M$ is as in Figure 24 2)-(4), the configuration (u,v, $\left.M_{L}\right)$ is $b a d$, and $\{u, v\} \neq\{b, c\}$. Choose an edge $x y \in M_{L}$ such that the configurations $\left(u, v, M_{L} \backslash\{x y\}\right)$ and $\left(x_{R}, y_{R}, M_{R}\right)$ are good. Observe in Figure 24 and Figure 1 that such an edge exists.

Thus there is a Hamiltonian path $P_{x_{R} y_{R}}$ of $Q_{R}-M_{R}$. Since the configuration $\left(u, v, M_{L}\right)$ is bad but the configuration $\left(u, v, M_{L} \backslash\{x y\}\right)$ is good, there is a Hamiltonian path $P_{u v}$ that contains edge $x y$ and avoids $M_{L} \backslash\{x y\}$. The desired Hamiltonian path of $Q_{4}-M$ is $P_{u x}+P_{x_{R} y_{R}}+P_{y v}$ where $P_{u x}$ and $P_{y v}$ are disjoint subpaths of $P_{u v}$, assuming that $x$ is closer to $u$ than $y$ on the path $P_{u v}$. This completes the proof of the lemma.

\section{Acknowledgements}

The authors are grateful to the anonymous referee whose constructive comments helped to improve the clarity of this paper. 


\section{References}

[1] R. CAHA, V. KOUBeK, Hamiltonian cycles and paths with a prescribed set of edges in hypercubes and dense sets, J. Graph Theory, 51-2 (2005), pp. 137-169.

[2] M. Y. CHAN, S-J. LEE, On the existence of Hamiltonian circuits in faulty hypercubes, SIAM J. Discrete Math., 4 (1991), pp. 511-527.

[3] T. DvořÁK, Hamiltonian cycles with prescribed edges in hypercubes, SIAM J. Discrete Math., 19 (2005), pp. 135-144.

[4] T. DVOřÁK, P. GREgOR, Hamiltonian paths with prescribed edges in hypercubes, Discrete Math., 307 (2007), pp. 1982-1998.

[5] J. FinK, Perfect matchings extend to Hamilton cycles in hypercubes, J. Combin. Theory Ser. B 97 (2007), pp. 1074-1076.

[6] R. ForCADE, Smallest maximal matchings in the graph of the d-dimensional cube, J. Combin. Theory Ser. B, 14 (1973), pp. 153-156.

[7] F. Gray, Pulse Code Communication, U.S. Patent 2,632,058, filed 13 November 1947, issued 17 March 1953.

[8] I. HAVEL, On Hamiltonian circuits and spanning trees of hypercubes, Čas. Pěst. Mat. 109 (1984), pp. 135-152.

[9] G. Kreweras, Matchings and Hamiltonian cycles on hypercubes, Bull. Inst. Combin. Appl. 16 (1996), pp. 87-91.

[10] F. T. Leighton, Introduction to Parallel Algorithms and Architectures: Arrays, Trees, Hypercubes, Morgan Kaufmann, San Mateo, CA, 1992.

[11] M. LeWInter, W. Widulski, Hyper-Hamilton laceable and caterpillar-spannable product graphs, Comput. Math. Appl., 34 (1997), pp. 99-104.

[12] F. Ruskey, C. SAVAGE, Hamilton cycles which extend transposition matchings in Cayley graphs of $S_{n}$, SIAM J. Discrete Math., 6 (1993), pp. 152-166.

[13] C. SaVAGE, A survey of combinatorial Gray codes, SIAM Rev., 39 (1997), pp. 605-629.

[14] C.-H. TsAI, Linear array and ring embeddings in conditional faulty hypercubes, Theor. Comput. Sci., 314 (2004), pp. 431-443.

[15] C.-H. Tsai, J. J. M. TAN, T. LiAng, L.-H. HsU, Fault-tolerant Hamiltonian laceability of hypercubes, Inf. Process. Lett., 83 (2002), pp. 301-306. 
